\title{
INCIDENCIA DE BIOFERTILIZANTES (Tetrabiol Y Lactobiol) CON ABONO QUÍMICO (PO5, KCl Y UREA) EN LAS VARIABLES FITOMETRICAS DE TRES GENOTIPOS DE MAIZ (Zea mays) (Criollo Mexicano, Hibrido DK 234 Y OGM-PIONNER 30F35H).
}

\section{INCIDENCE OF BIOFERTILIZERS (Tetrabiol and Lactobiol) WITH CHEMICAL FERTILIZER ( $\mathrm{PO}_{5}, \mathrm{KCl}$ and UREA) IN THE PHYTOMETRIC VARIABLES OF THREE MAIZ GENOTYPES (Zea mays) (Mexican criollo, Hybrid DK 234 and OGM-PIONNER 30F35H).}

\author{
Socarras B. Julio C. ${ }^{1,3}$, Ortega S. Yina P. ${ }^{2}$, Tafur Jesús. O. ${ }^{2}$,Orozco O José A. ${ }^{1,3}$ \\ ${ }^{1}$ Universidad Popular del Cesar Aguachica. GIPTA. jcsocarras@misena.edu.co \\ ${ }^{2}$ Ingeniería Agroindustrial, Universidad Popular del Cesar Aguachica \\ ${ }^{3}$ Servicio Nacional de Aprendizaje SENA. BIOSENA. Instructor SENNOVA. jaorozco@ sena.edu.co
}

\section{Resumen}

Desde su origen el maíz ha sido fundamental para los seres humanos, contribuye a en gran medida a la economía colombiana, su cultivo es muy importante en el sur del Cesar, aunque ha disminuido en los últimos años por la sequía y otros aspectos. El maíz ha sufrido alteraciones genéticas y cruce de variedades para incrementar su producción. Se evaluó el comportamiento de las variables fitométricas de la mazorca (grano, tusa, capacho y hojas), rendimiento por hectárea, altura máxima, altura final en tres (3) genotipos de maíz (Criollo Mexicano, hibrido DK 234 y O.G.M-Pionner 30F35H), utilizando Biofertilizantes combinados con Nitrógeno, Fosforo y Potasio (NPK), con adición de microorganismos solubilizadores de Fosforo y fijadores de Nitrógeno. Las características fitométricas del maíz en su mayoría no presentaron significancia, solo presentaron discrepancias en relación a los genotipos y el peso del grano, donde el maíz modificado genéticamente (OGM Pionner 30F35H), presentó mayor peso promedio $(115,8725+/-13,2405) \mathrm{gr}$. Seguido del hibrido DK (81,2375+/- 9,2459 gr), el de menor peso fue el Criollo Mexicano $(79,8075+/-3,5778$ gr). Respecto al rendimiento de la producción la variedad OGM fue la mejor (6,4 T/Ha promedio), esta posee características específicas para proveer un desarrollo eficaz, el Criollo Mexicano mostró menor rendimiento 4,45 T/ Ha. El tratamientos de maíz que presentó una mayor producción fue el fertilizado con NPB con 5,2 T/Ha y el de menor rendimiento fue el NPK testigo con 4,9333 $\mathrm{T} / \mathrm{Ha}$. debido a que estos nutrientes son esenciales para determinar la cantidad de materia seca total..

Palabras clave: Fertilizante, fotométricas, genotipo, maíz.

Abstract

Since its origin, corn has been fundamental for human beings, contributes greatly to the Colombian economy, its cultivation is very important in the south of Cesar, although it has decreased in recent years due to drought and other aspects. The corn has undergone genetic alterations and varieties crossing to increase its production. We evaluated the behavior of the variables of the ears (grain, tusso, layer and leaves), yield per hectare, maximum height, final 
height in three (3) genotypes of corn (Criollo Mexicano, hybrid DK 234 and OGM-Pionner 30F35H ), using Biofertilizers combined with Nitrogen, Phosphorus and Potassium (NPK), with addition of Phosphorus solubilizing microorganisms and Nitrogen fixatives. The phytomedic characteristics of maize were not significant, only showed discrepancies in relation to genotypes and grain weight, where genetically modified maize (GMO Pionner $30 \mathrm{~F} 35 \mathrm{H}$ ), presented higher average weight (115.8725 +/$13,2405)$ gr. Following the hybrid DK (81.2375 $+/-9.2459$ gr), the one with the lowest weight was the Mexican Criollo (79.8075 +/- 3.5778 gr).

\section{Introducción}

El maíz (Zea mays) es un cultivo importante en Colombia, donde se adapta en varias regiones con diferentes condiciones agroclimáticas y socioeconómicas. El grano se produce desde la Guajira hasta el Amazonas y desde la vertiente del Pacífico hasta los Llanos Orientales; se siembra desde el nivel del mar hasta 3000 m.s.n.m., en zonas con precipitaciones menores que $300 \mathrm{~mm}$ en la Guajira hasta 10,000 mm en el Choco. Los tipos blanco y amarillo son los más cultivados, el primero se dedica al consumo humano y el segundo principalmente a usos industriales en la elaboración de concentrados para cría y engorde de animales.(Rodríguez, 2015). En estos tipos de cultivos se utilizan insumos químicos como los fertilizantes, los pesticidas, herbicidas, venenos, entre otros. (Henry, Zarta, Rivera, \& Sanchez, 2016).Lograr la sincronía entre el suministro de $\mathrm{N}$ y la demanda de los cultivos sin causar deficiencia es la clave para optimizar la comercialización considerando la productividad del cultivo, las ganancias y la calidad ambiental. Los sistemas de producción de cereales (trigo, maíz, arroz, cebada, sorgo, mijo, avena, y centeno) la eficiencia del uso del nitrógeno (NUE) es aproximadamente $33 \%$. El $67 \%$ representa pérdidas de los fertilizantes nitrogenados a través
Regarding the yield of the production, the OGM variety was the best (6.4 $\mathrm{T} / \mathrm{Ha}$ average), this has specific characteristics to provide an effective development, the Mexican Criollo showed lower yield $4.45 \mathrm{~T} / \mathrm{Ha}$. The corn treatments that presented a higher production was fertilized with NPB with 5.2 T / Ha and the lowest yield was the control NPK with $4.9333 \mathrm{~T} / \mathrm{Ha}$. Because these nutrients are essential to determine the amount of.

Keywords: Corn, fertilizer, genotype, photometric.

de escorrentías y volatilización. Este hecho representa un gran problema para la mayoría de cultivos en general. (Vergara Rodríguez, Diaz Rodriguez, \& Alvarez, 2017).Arias en 2010 plantea que el uso de agroquímicos además de ser de alto costo, hace que el suelo pierda diversidad de flora, fauna y que se destruya su materia orgánica, mientras que los EM mejoran la biota del suelo, sus propiedades físicas, disminuyen los costos de la producción, aumenta la cosecha y los ingresos del agricultor.(Arias Hoyos, 2010)Algunos de los efectos benéficos de la aplicación de los EM son: a) Promueve la germinación, la floración, el desarrollo de los frutos y la reproducción de las plantas. b) Mejora física, química y biológicamente el ambiente de los suelos y suprime los patógenos que promueven enfermedades. c) Aumenta la capacidad fotosintética de los cultivos. d) Asegura una mejor germinación y desarrollo de las plantas. e) Incrementa la eficacia de la materia orgánica como fertilizante. f) Reduce los malos olores y se disminuye la utilización de desinfectantes. g) Disminuye el agua de lavado, implementando el manejo de camas secas para colectar excretas. h) Ayuda al aprovechamiento eficiente de desechos animales. i) Mejora la calidad y aumenta la rapidez en la elaboración del abono entre otros beneficios (Luna Feijoo \& Mesa Reinaldo, 2016) 
El uso de variedades mejoradas, así como biofertilizantes elaborados con hongos micorriza favorecen la nutrición y permiten complementar el uso de fertilizantes químicos, lo que podría incrementar la producción.(Tadeo Robledo \& etal, 2016) Se han usado altas cantidades de nitrógeno químico $(\mathrm{N})$ en todo el mundo para aumentar los rendimientos de los cultivos. Aproximadamente 23 millones de toneladas de $\mathrm{N}$ fertilizantes se aplicaron en 2008 en China, lo que representa alrededor del $30 \%$ del consumo mundial total de N (Duan et al., 2014).

Se evaluó el comportamiento de las variables fitométricas de la mazorca (grano, tusa, capacho y hojas), rendimiento por hectárea, altura máxima, altura final en tres (3) genotipos de maíz (Criollo Mexicano, hibrido DK 234 y O.G.MPionner 30F35H), utilizando Biofertilizantes combinados con Nitrógeno, Fosforo y Potasio (NPK), con adición de microorganismos solubilizadores de Fosforo y fijadores de Nitrógeno.

\section{Metodología}

La investigación se realizó en el corregimiento de montecitos del municipio de rio de oro, en un lote de $2500 \mathrm{~m}^{2}$ dedicado tradicionalmente a la producción de maíz. Se utilizaron 3 genotipos de maíz: DK247R, Pionner 30F35 y Una variedad Criolla conocida en la región como Criollo Mexicano. Se sembro a una distancia entre planta de $0,2 \mathrm{~m}$. 0,9 $\mathrm{m}$ entre surcos y una longitud de 6 m. resultando alrededor de 55500 planta.ha $^{-1}$. El diseño experimental fue de bloques completos al azar en arreglo factorial $4 \times 3$, definiendo 3 tratamientos; Fertilización de Fosforo (T1=PB), Fertilización combinada con Fijadores de Nitrógeno $(\mathrm{T} 2=\mathrm{NB})$ un último tratamiento que fue una mezcla de los tres (3) $(\mathrm{T} 3=\mathrm{NPK}+\mathrm{NPB})$. En cada uno se realizó un reemplazo del $30 \%$ de la fertilización comercial (NPK) a los que se agregó una fertilización tradicional $(\mathrm{T} 0=\mathrm{NPK})$ como parámetro de referencia, todo esto en cuatro repeticiones por tratamiento. A cada parcela se le realizaron 13 muestreos de Altura de la planta, caracterización de la mazorca (Peso de grano, Peso de capacho, Peso de tusa).

\section{Análisis estadístico}

En el análisis estadístico se aplicó análisis de varianza (ANOVA) y se aplicaron las pruebas POSTHOC de Tukey $(\mathrm{p} \leq 0,05)$ usando el software SPSS v. 23.

\section{Resultados}

La evaluación agronómica de la incorporación del producto biofertilizante fue cuantificada a través de la respuesta del cultivo en las siguientes variables fitométricas y de rendimiento. Una vez realizado el desgrane, se procedió a tomar los pesos de los granos, y realizar comparaciones en rendimiento por genotipo permitiendo evaluar el efecto de los biofertilizantes sobre el llenado de los granos.

Tabla 1: Resultados de las comparaciones del promedio peso de grano por genotipo

\begin{tabular}{|l|c|}
\hline GENOTIPOS & $\begin{array}{l}\text { PROMEDIO PESO DE } \\
\text { GRANO (g) }\end{array}$ \\
\hline Criollo Mexicano & $\mathbf{7 9 , 8 0 8}+/-3,578$ \\
\hline hibrido DK 234 & $\mathbf{8 1 , 2 3 8 + / - ~ 9 , 2 4 6}$ \\
\hline OGM-Pionner 30F35H & $115,873+/-\mathbf{1 3 , 2 4 1}$ \\
\hline
\end{tabular}

Fuente: Los investigadores

En cuanto al peso del grano de acuerdo al el genotipo, el maíz modificado genéticamente, presenta un peso superior frente a los otros dos genotipos; el maíz hibrido y el criollo no difiere en gran medida. El desarrollo de estos organismos (OGM) ha crecido vertiginosamente, considerándose hoy en día la tecnología biotecnológica de más rápida adopción y crecimiento por área sembrada a nivel global (Castaño-Hernández, 2015).

23 
Grafico 1. Peso promedio de granos por genotipo

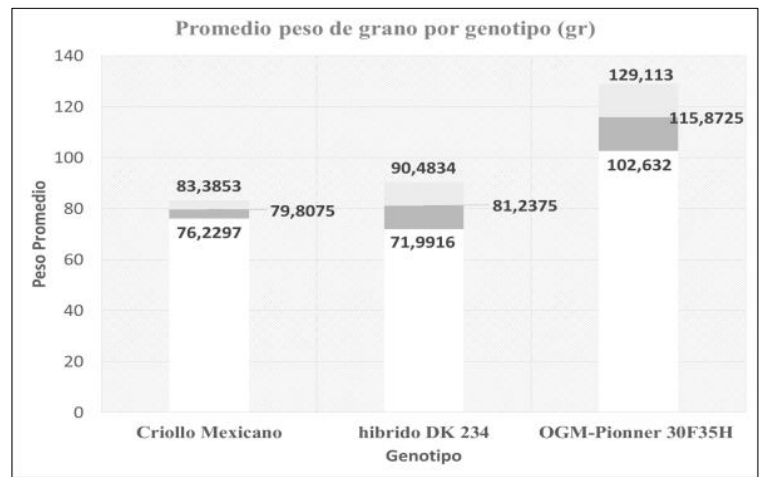

Fuente: Los investigadores

Se encontraron diferencias significativa entre los genotipos (Hibrido, Criollo y O.G.M), con un $\mathrm{p}$ valor de $1 \%$ a un nivel de confianza del $95 \%, 2$ grados de libertad y $5 \%$ de error. Según (TRIOLA,2009) el O.G.M presenta un mejor rendimiento en cuanto al peso del grano, alejándose de forma marcada de los resultados obtenidos en los otros dos genotipos; debido al mejoramiento genético que posee, permitiéndole ser más resistente a enfermedades y herbicidas en comparación al maíz hibrido y criollo. De acuerdo a los resultados se rechaza la hipótesis nula. El rendimiento del maíz grano, ton/ha, producido en distritos de riego es igual al de cultivo normal (temporal) con una probabilidad de $95 \%$, y que la producción en temporal tiene un menor costo social y ambiental, por lo menos en términos de agua (Montesillo-Cedillo, 2016)

Tabla 2: Resultados de las comparaciones del promedio peso de grano por Tratamiento (Fertilizante)

\begin{tabular}{|c|c|}
\hline $\begin{array}{c}\text { TRATAMIENTO } \\
\text { (Fertilizante) }\end{array}$ & $\begin{array}{c}\text { PROMEDIO PESO DE } \\
\text { GRANO }(\mathrm{g})\end{array}$ \\
\hline PB & $\mathbf{9 3 , 7 7 7 + / - 3 2 , 2 1 2}$ \\
\hline NPB & $95,238+/-\mathbf{1 8 , 1 5 3}$ \\
\hline NB & $91,160+/-7,798$ \\
\hline NPK & $\mathbf{8 9 , 0 5 0 + / - 2 3 , 4 7 9}$ \\
\hline
\end{tabular}

Fuente: Los investigadores

A rasgos generales que el tratamiento NPB, PB y NB no muestran gran diferencia en cuanto al peso del grano, pero existe una tendencia hacia el tratamiento NPB con peso en grano igual a 95,238 gramos (tabla 2).

Grafico 2. Peso promedio de granos por fertilizante

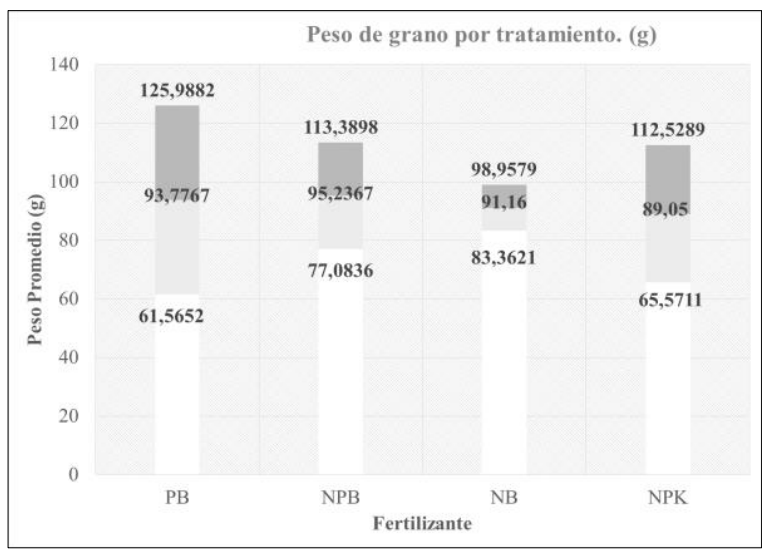

Fuente: Los investigadores

En relación a los tratamientos que no existe diferencia significativa entre ellos, por lo tanto se acepta la hipótesis nula, con un margen de error del $5 \%$, p valor de $98,7 \%$ para un nivel de confianza del $95 \%$ y grados de libertad 3. Existe una tendencia hacia la fertilización con solubilizador de fosforo y fijador de nitrógeno, la planta realiza una mejor absorción de nutrientes con la ayuda de los biológicos; que permite obtener un leve incremento en peso del grano respecto a los otros tratamientos.

Tabla 3: Resultados de las comparaciones del promedio peso del capacho por genotipo.

\begin{tabular}{|c|c|}
\hline GENOTIPO & $\begin{array}{c}\text { PROMEDIO PESO DE } \\
\text { CAPACHO }(\mathrm{g})\end{array}$ \\
\hline Criollo & $27,858+/-\mathbf{3 , 4 8 7}$ \\
\hline Hibrido DK234 & $\mathbf{1 8 , 5 6 3 + / - 3 , 9 7 6}$ \\
\hline OGM 30F35H & $\mathbf{2 2 , 1 7 8 + / - 3 , 4 9 5}$ \\
\hline
\end{tabular}

Fuente: Los investigadores

En la Tabla 3 se puede observar el comportamiento del capacho en relación a su peso respecto a los diversos genotipos, encontrando ciertas diferencias entre cada uno, siendo así el maíz criollo el que mayor peso registró $(27,8575$ gramos). 
Se encontró diferencias significativas entre los genotipos, motivo por el cual la hipótesis nula es rechazada, tomando como margen de error $5 \%, \mathrm{p}$ valor de $1,8 \%$ sobre el porcentaje de confianza que es del $95 \%$ con 2 grados de libertad. El maíz criollo desarrolló un mayor peso en capacho, en relación al maíz hibrido y O.G.M; un peso superior en capacho no es favorable a nivel comercial, en el mercado se toma en cuenta es el peso en grano y lo que se pretende es obtener plantas que desarrollen mazorcas con más hileras, tusa delgada y menor peso en capacho. Es por ello que para este caso el maíz Hibrido con un peso inferior a los otros dos genotipos (18,563 gramos) es una respuesta positiva a nivel comercial.

Tabla 4: Resultados de las comparaciones del promedio peso del capacho por tratamiento (Fertilizante)

\begin{tabular}{|c|c|}
\hline $\begin{array}{c}\text { TRATAMIENTO } \\
\text { (Fertilizante) }\end{array}$ & $\begin{array}{c}\text { PROMEDIO PESO DE } \\
\text { CAPACHO }(\text { g) }\end{array}$ \\
\hline PB & $22,083+/-7,132$ \\
\hline NPB & $24,273+/-2,836$ \\
\hline NB & $3,589+/-21,690$ \\
\hline NPK (Testigo) & $\mathbf{8 , 3 6 9 + / - ~ 2 3 , 4 1 7}$ \\
\hline
\end{tabular}

Fuente: Los investigadores

Se puede observar en la Tabla 4 que los tratamientos presentan resultados cercanos, obteniendo que el tratamiento NPB muestra un mayor peso en capacho (24,273 gramos) en comparación a los otros tratamientos. El comportamiento del capacho en relación a su peso respecto a los diversos tratamientos aplicados no mostro diferencia significativa, con $\mathrm{p}$ valor de 94,3\% además un nivel de confianza del $95 \%$ con margen de error del 5\%; en términos generales la hipótesis nula es aceptada.

En la Tabla 5 muestra que el rendimiento total en peso de la tusa difiere respecto al genotipo, encontrando que el maíz hibrido presenta cierta relación con el maíz criollo, mientras que el maíz modificado genéticamente arroja un peso superior, existe diferencia significativa entre los genotipos en cuanto al peso de la tusa lo que permite rechazar la hipótesis nula.

Tabla 5: Comparación de los promedio en cuanto al peso de la tusa por genotipo.

\begin{tabular}{|c|c|}
\hline GENOTIPO & $\begin{array}{c}\text { PROMEDIO PESO DE } \\
\text { TUSA }(\mathrm{g})\end{array}$ \\
\hline Criollo & $35,740+/-\mathbf{3 , 0 6 5}$ \\
\hline Hibrido DK234 & $\mathbf{3 6 , 7 0 8}+/-\mathbf{3 , 8 1 1}$ \\
\hline OGM 30F35H & $\mathbf{4 4 , 9 8 0 + / - 3 , 0 5 4}$ \\
\hline
\end{tabular}

Fuente: Los investigadores.

En relación a los tratamientos se puede deducir que existe una tendencia hacia la fertilización PB, puesto que fue el tratamiento que tuvo un leve incremento en cuanto al peso de tusa $(39,467$ gramos), y el que menor rendimiento presentó fue el tratamiento testigo (NPK) con un peso de 38,910 gramos, todos los tratamientos presentaron resultados similares por lo que no se encontraron diferencias significativas. El reconocimiento de estos dos efectos algo diferentes (es decir, aumentar las tasas de crecimiento y las etapas avanzadas de crecimiento) en el crecimiento del maíz podría ayudar a definir la deficiencia de $\mathrm{N}$ con mayor precisión y mejorar la precisión del diagnóstico del estado de $\mathrm{N}$ en la agricultura de producción (Zhang, Blackmer, Kyveryga, Van De Woestyne, \& Blackmer, 2008).

Tabla 6: Resultados de las comparaciones del promedio peso del Tusa por tratamiento (Fertilizante)

\begin{tabular}{|l|c|}
\hline $\begin{array}{c}\text { TRATAMIENTO } \\
\text { (Fertilizante) }\end{array}$ & $\begin{array}{c}\text { PROMEDIO PESO } \\
\text { DE TUSA (g) }\end{array}$ \\
\hline PB & $39,467+/-7,222$ \\
\hline NPB & $38,847+/-7,438$ \\
\hline NB & $39,348+/-2,128$ \\
\hline NPK (Testigo) & $\mathbf{3 8 , 9 1 0 + / - ~ 6 , 3 7 2}$ \\
\hline
\end{tabular}

Fuente: Los investigadores 
Tabla 7 Resultados de las comparaciones del promedio peso de la mazorca por genotipo.

\begin{tabular}{|l|c|}
\hline GENOTIPO & $\begin{array}{c}\text { PROMEDIO PESO DE } \\
\text { MAZORCA }(\mathrm{g})\end{array}$ \\
\hline Criollo & $143,403+/-4,053$ \\
\hline Hibrido DK234 & $136,508+/-16,934$ \\
\hline OGM 30F35H & $183,028+/-18,877$ \\
\hline
\end{tabular}

Fuente: Los investigadores

La variabilidad del peso de la mazorca respecto a los genotipos estudiados, se observa que el maíz O.G.M presenta un mayor peso en mazorca; (183,028 gramos), seguido del maíz criollo con 143,403 gramos y en última instancia con un peso inferior de 136,508 gramos se encuentra el maíz hibrido.

Tabla 8: Resultados de las comparaciones del promedio peso de la mazorca por tratamiento (Fertilizante)

\begin{tabular}{|l|c|}
\hline $\begin{array}{c}\text { TRATAMIENTO } \\
\text { (Fertilizante) }\end{array}$ & $\begin{array}{c}\text { PROMEDIO PESO DE } \\
\text { MAZORCA }(\mathrm{g})\end{array}$ \\
\hline PB & $155,327+/-43,021$ \\
\hline NPB & $158,350+/-25,869$ \\
\hline NB & $152,197+/-6,075$ \\
\hline NPK (Testigo) & $151,377+/-30,133$ \\
\hline
\end{tabular}

Fuente: Los investigadores

En relación a los tratamientos se puede observar valores relativamente cercanos en cuanto a pesos de mazorca como se muestra en la tabla 8; en donde el maíz fertilizado con NPB presenta un mayor peso de mazorca que equivale a 158,35 gramos y el menor rendimiento fue el tratamiento testigo con unos 151,377 gramos.

Tabla 9: Comparaciones del promedio de la altura máxima por genotipo.

\begin{tabular}{|l|c|}
\hline GENOTIPO & $\begin{array}{l}\text { PROMEDIO ALTURA } \\
\text { (Cm) Por genotipo }\end{array}$ \\
\hline Criollo & $199,300+/-15,162$ \\
\hline Hibrido DK234 & $187,275+/-0,386$ \\
\hline OGM 30F35H & $201,950+/-9,466$ \\
\hline
\end{tabular}

Fuente: Los investigadores
La tabla 9 muestra que la altura máxima de la planta varía respecto al genotipo, de la cual se puede observar que el O.G.M presenta una mayor altura equivalente a $201.950 \mathrm{~cm}$ sin diferencias reveladoras entre cada genotipo; mientras que el maíz hibrido muestra un menor rendimiento con una altura en promedio de $187,275 \mathrm{~cm}$.

Tabla 10: Resultados de las comparaciones de la altura máxima por tratamiento (Fertilizante)

\begin{tabular}{|l|c|}
\hline $\begin{array}{c}\text { TRATAMIENTO } \\
\text { (Fertilizante) }\end{array}$ & $\begin{array}{c}\text { PROMEDIO PESO DE } \\
\text { MAZORCA (g) }\end{array}$ \\
\hline PB & $193,033+/-14,197$ \\
\hline NPB & $193,100+/-7,351$ \\
\hline NB & $194,967+/-11,558$ \\
\hline NPK (Testigo) & $203,600+/-14,868$ \\
\hline
\end{tabular}

Fuente: Los investigadores

Respecto a los tratamientos se puede observar en la tabla 10 que todos los tratamientos respondieron de forma similar, excepto el maíz fertilizado con tratamiento testigo con una altura en promedio de 203,600 cm.

\section{Conclusiones}

El rendimiento en producción en términos generales se presenta en mejor forma con el uso de maíz O.G.M, con reemplazo del $30 \%$ en la fertilización por solubilizador de fosforo y fijador de nitrógeno (15\% respectivamente); debido a que estos nutrientes son esenciales para determinar la cantidad de materia seca total, conociendo que el fosforo por sí solo no puede ser absorbido en gran proporción por la planta, solo el $12 \%$ del fosforo en la planta proviene del fertilizante.

Con la mezcla de $\mathrm{N}$ y $\mathrm{P}$ en los fertilizantes, se logran dos objetivos: incrementa la cantidad de $\mathrm{P}$ que entra a la planta y mejora la eficiencia del fertilizante, al conocer que utilizando una fuente más eficiente en la absorción de $\mathrm{P}$ por la planta, los kilogramos necesarios serán menores pero la asimilación de fósforo será mayor, repercutiendo 
en el rendimiento final de la materia seca determinando la producción.

Los materiales estudiados presentaron un porte similar, sin embargo en relación al peso de granos en mazorca, peso del capacho y peso de la tusa se presentaron discrepancias notables en donde el OGM, presentó un mayor peso promedio de granos en mazorca con 115,873 gramos, el genotipo criollo sobresalió con un peso de capacho promedio de 27,858 gramos y por último en la variable peso de la tusa resalta el O.G.M con un peso 44,980 gramos. En promedio la mazorca de mayor peso promedio fue el OGM, en las demás variables, los resultados no variaron en gran término.

\section{Agradecimientos}

Universidad Popular Del Cesar Seccional Aguachica y Grupo de Investigación GIPTA.

Servicio Nacional De Aprendizaje Sena. Grupo de Investigación Biosena.

\section{Referencias Bibliograficas}

Arias Hoyos, A. (2010). MICROORGANISMOS EFICIENTES Y SU BENEFICIO PARA LA AGRICULTURA Y EL MEDIO AMBIENTE. JOURNAL DE CIENCIA E INGENIERÍA, 4245.

Castaño-Hernández, A. (2015). Alimentos derivados de cultivos genéticamente modificados. ¿Nuevos, seguros para la salud humana, consumidos? Pediatría, 48(3), 68-74. doi: https://doi.org/10.1016/j.rcpe.2015.09.001

Duan, Y., Xu, M., Gao, S., Yang, X., Huang, S., Liu, H., \& Wang, B. (2014). Nitrogen use efficiency in a wheat-corn cropping system from
15 years of manure and fertilizer applications. Field Crops Research, 157, 47-56. doi: https://doi.org/10.1016/j.fcr.2013.12.012.

Henry, H. A. Z. A., Zarta, A., Rivera, M. E., \& Sanchez, L. R. (2016). Evaluación de las áreas estratégicas en Pamplona. BISTUA REVISTA DE LA FACULTAD DE CIENCIAS BASICAS, 14(1), 47-61. https://doi.org/10.24054/01204211.v1.n1.2016.1 937

Luna Feijoo, M. A., \& Mesa Reinaldo, J. R. (2016). Microorganismos eficientes y sus beneficios para los agricultores. Agroecosistemas.

Montesillo-Cedillo, J. L. (2016). Rendimiento por hectárea del maíz grano en México: distritos de riego vs temporal. Economía Informa, 398, 60-74. doi: https://doi.org/10.1016/j.ecin.2016.04.005

Rodríguez, C. P. (2015). Produccion de semilla y cruzamientos entre accesiones de maíz del deparrtamento del Magdalena, Colombia. Acta Agronómica, 64.

Tadeo Robledo, M., \& etal. (2016). Biofertilización en híbridos de maíz androestériles y fértiles para los Valles Altos de México. Terra Latinoamericana, 65 - 72.

TRIOLA, Mario F. Estadística. Impreso por PEARSON. México D.F. 2009. P. 634 - 642.

Vergara Rodríguez, D. R., Diaz Rodriguez, G., \& Alvarez, Á. P. (2017). Efecto de la Fertilización Nitrogenada en el Cultivo de Maíz para la Caracterización de la Emisión de Gases Efecto Invernadero. Documentos de Trabajo.

Zhang, J., Blackmer, A. M., Kyveryga, P. M., Van De Woestyne, B. W., \& Blackmer, T. M. (2008). 
Fertilizer-Induced Advances in Corn Growth Stage and Quantitative Definitions of Nitrogen Deficiencies. Pedosphere, 18(1), 60-68. doi: https://doi.org/10.1016/S1002-0160(07)60103-9

\section{RESEÑA AUTORES}

Julio Cesar Socarras Ballesta; Ingeniero Agroindustrial (2004) Universidad Popular del Cesar; Especialista en Administración de la Informática Educativa, Estudiante de Maestría en Nutrición y Biotecnología Alimentaria; Instructor Agroindustria Servicio Nacional de Aprendizaje Sena; Docente Catedrático Universidad Popular del Cesar, Investigador Grupo Gipta (UPC) y Grupo BIOSENA (Centro Agroempresarial SENA Aguachica) https://orcid.org/0000-0002$\underline{5835-830 X}$

Jose Alejandro Orozco Ochoa; Ingeniero Agroindustrial (2004) Universidad Popular del Cesar; Especialista en Administración de la Informática Educativa, Estudiante de Maestría Ciencia Y Tecnología de Alimentos Un pamplona; Instructor Investigador SENNOVA Servicio Nacional de Aprendizaje Sena; Docente Asociado - Catedrático Universidad Popular del Cesar, Investigador Grupo de investigación Grupo BIOSENA (Centro Agroempresarial SENA Aguachica) y del grupos de investigación Gipta (Universidad Popular del Cesar) https://orcid.org/0000-0001-6505-0234

Gina Paola Ortega Santiago; Ingeniera Agroindustrial Universidad Popular del Cesar. Estudiante Maestría En Universidad De Los Andes.

Jesús Orlando Tafur Chinchilla; Ingeniera Agroindustrial Universidad Popular del Cesar.

*Para citar este artículo: Socarras B. JC, OrtegaS. YP., Tafur JO.,Orozco O JA. Incidence of Biofertilizers (Tetrabiol and Lactobiol) with chemical fertilizer ( $\mathrm{PO}_{5}, \mathrm{KCl}$ and UREA) in the Phytometric variables of three maíz genotypes (Zea mays) (Mexican criollo, Hybrid DK 234 and OGM-PIONNER 30F35H). Revista Bistua.2017.15(2):03-10

+ Autor para el envió de correspondencia y la solicitud de las separatas: Socarras B. JC Universidad Popular del Cesar Aguachica. GIPTA.jcsocarras@misena.edu.co

Recibido: Octubre 05 de 2016

Aceptado: Enero 29 de 2017 